\title{
Is it time to revisit tracheal intubation for Cesarean delivery?
}

\author{
Ashraf S. Habib, MBBCh
}

Received: 29 March 2012/Accepted: 13 April 2012/Published online: 24 April 2012

(C) Canadian Anesthesiologists' Society 2012

Significant progress has been achieved in obstetric anesthesia. Most notably, there has been a dramatic reduction in anesthesia-related maternal mortality. The Confidential Enquiries into Maternal Deaths in the United Kingdom was established in 1952, and in each of the first ten triennial reports, 30-50 deaths were attributed directly to anesthesia. ${ }^{1}$ In contrast, the last eight reports spanning 24 years included 41 direct anesthetic deaths, ${ }^{2}$ almost the same number that occurred over each three-year period in the earlier reports. A similar picture was also seen in North America. This reduction in anesthesia-related mortality is even more impressive considering the increasing number of anesthetics administered to parturients and the continuing rise in the rate of Cesarean delivery (CD) over the past 60 years. In the UK, it has been estimated that anesthesia for CD was more than 30 times safer in 2002 than in 1964 . $^{1}$

There are two main reasons for this remarkable success story. First, general anesthesia for CD has become much safer, and second, we have largely avoided general anesthesia by adopting neuraxial techniques as standard practice for the majority of CDs. The leading cause of death due to general anesthesia is related to airway management. Initially, aspiration of gastric contents was the major factor resulting in maternal mortality. A number of changes were introduced over several years to address this problem, including the technique of rapid sequence induction with cricoid pressure, avoidance of mask ventilation before tracheal intubation, and use of sodium citrate and $\mathrm{H}_{2}$ blockers. However, the requirement for tracheal

\footnotetext{
A. S. Habib, MBBCh $(\square)$

Department of Anesthesiology, Duke University Medical Center, Box 3094, Durham, NC 27710, USA

e-mail: habib001@dm.duke.edu
}

intubation for CD in the 1960s introduced another problem, i.e., the emergence of failed tracheal intubation and inadequate oxygenation as factors leading to 34 maternal deaths in the UK over a nine-year period from 1976 to $1984 .{ }^{1}$ Such fatal events have since become unusual as a result of improved training, better monitoring with the use of oximetry and capnography, and improved airway management with the introduction of failed intubation drills and the increasing availability of airway rescue devices.

The Laryngeal Mask Airway ${ }^{\mathrm{TM}}$ (LMA) is the main rescue device incorporated in failed tracheal intubation drills. Case reports and case series have highlighted its successful use after failed tracheal intubation in CDs. ${ }^{3,4} \mathrm{In}$ 2001, Han reported the use of the LMA Classic ${ }^{\mathrm{TM}}$ in 1,067 parturients undergoing elective $\mathrm{CD} .^{5}$ More recently, Halaseh et al. described their experience using the LMA ProSeal $^{\mathrm{TM}}$ (PLMA) in 3,000 women undergoing the same procedure. ${ }^{6}$ Both studies reported no cases of aspiration, but there was one case of regurgitation. ${ }^{6}$ In this issue of the Journal, Yao et al. add to this literature and report the use of the LMA Supreme ${ }^{\text {TM }}$ (SLMA) in 700 Chinese parturients. ${ }^{7}$ They carefully selected low-risk patients for inclusion in their study. Women fasted for at least six hours for elective cases and four hours for urgent cases, and they were excluded if they had a potentially difficult airway, a body mass index $(\mathrm{BMI}) \geq 35 \mathrm{~kg} \cdot \mathrm{m}^{-2}$, or gastroesophageal reflux. Two providers experienced in the use of the SLMA inserted the airway device in all patients following induction of anesthesia and muscle relaxation with rocuronium. Antacid prophylaxis was achieved with oral sodium citrate and ranitidine. Cricoid pressure was released after confirming adequate ventilation and inflation of the SLMA cuff. A pre-mounted orogastric tube was used to aspirate gastric contents after insertion of the SLMA and before 
emergence. The insertion of the SLMA was successful in all cases (98\% on first attempt and $2 \%$ on second attempt). Gastric tube placement was also successful in all cases and aspirated a mean of $25 \mathrm{~mL}$ of gastric secretions with a mean $\mathrm{pH}$ of 2.2. There were no detectable leaks, no episodes of desaturation to $<92 \%$, and no signs of laryngospam, bronchospasm, or evidence of regurgitation or aspiration. Fewer than $4 \%$ of parturients reported sore throat, and there was no difference in outcomes between elective (576 cases) and urgent (124 cases) CDs.

The study was well conducted, but the report lacks some useful details. For instance, the authors did not report hemodynamic changes associated with insertion of the SLMA, which could be an advantage compared with tracheal intubation. Some authors have suggested that cricoid pressure could impede insertion and ventilation with the LMA, ${ }^{8}$ but Yao et al. did not state if this problem was encountered or if cricoid pressure had to be released to facilitate insertion and proper positioning of the SLMA. The $\mathrm{pH}$ of gastric aspirate was low despite antacid prophylaxis; this could be related to suboptimal timing of administration, which was not specified by the authors. The traditional definition of aspiration risk (gastric acid $>25 \mathrm{~mL}$, or $0.4 \mathrm{~mL} \cdot \mathrm{kg}^{-1}$, and $\mathrm{pH}<2.5$ ) would suggest that even some of those slim fasted parturients were at risk. While some authors have argued for the use of rocuronium instead of succinylcholine for $\mathrm{CD},{ }^{9}$ the dose used by Yao et al. is lower than that advocated for rapid sequence induction in this patient population, especially where sugammadex is available, ${ }^{10}$ so a slower onset time compared with succinylcholine would be expected. This choice might have been influenced by the short duration of the procedure in their practice. This lower dose might also be adequate to prevent coughing and bucking during SLMA insertion before providing adequate intubating conditions, but Yao et al. did not provide information on the time from induction to SLMA insertion. It is also not clear if the women having urgent $\mathrm{CDs}$ were in labour or received opioid analgesics, which are known to slow gastric emptying.

How should we interpret the findings of this study? Do these findings apply to our practice? Indeed, we need to highlight significant differences between CDs performed in this study and those performed under general anesthesia in North America and many parts of Europe and Australia. For instance, general anesthesia is commonly employed in Yao's institution, accounting for $83 \%$ of CDs performed during the one-year period of the study. This is in stark contrast to our current practice where general anesthesia is reserved for some emergency situations or in cases where there is a failed block or contraindication to regional anesthesia, such as coagulopathies. Some centres have reported general anesthesia rates of less than $1 \%{ }^{11}$
Therefore, our patients might not fast for four to six hours as in Yao et al.'s study. They are also likely to be in pain and might have received opioid analgesics, factors known to increase the risk of having a "full stomach". Patient demographics in our practice are also very different, with an increasing prevalence of obesity. In Yao et al.'s study, the mean weight of patients was $65 \mathrm{~kg}$ with a BMI of $26 \mathrm{~kg} \cdot \mathrm{m}^{-2}$, whereas women undergoing $\mathrm{CD}$ at my institution have a mean BMI of $33 \mathrm{~kg} \cdot \mathrm{m}^{-2} \cdot{ }^{12}$ The authors pointed out that the obstetricians were instructed to avoid excessive fundal pressure. In my practice, excessive fundal pressure is commonly applied during delivery, and I am not sure if our obstetricians can predictably avoid this practice. Fundal pressure can lead to regurgitation and, therefore, could be a risk for aspiration. In fact, the case of regurgitation in the previous case series involving the use of a PLMA occurred with fundal pressure at delivery. ${ }^{6}$ In the study in this issue of the Journal, the SLMA was inserted by two experienced providers under optimal conditions with muscle relaxation, and the correct positioning was confirmed. Accordingly, those findings might not translate to cases where the SLMA is inserted by less experienced providers, a common situation in our teaching institutions. The recently published audit of the Royal College of Anaesthetists and the Difficult Airway Society of the UK cited obesity and use by junior providers as factors involved in major airway complications with supraglottic devices. ${ }^{13}$ In Yao et al.'s study, the duration of the surgical procedure was also shorter than that seen in many teaching institutions. Therefore, the patient population and practice in this Chinese hospital are very different from North American practice where women receiving general anesthesia for $\mathrm{CD}$ present a much higher risk for regurgitation and aspiration.

The SLMA was introduced in 2007 as a modified singleuse version of the PLMA. Studies have reported that success rate, insertion time, and complications are comparable between these two devices, but the oropharyngeal leak pressure is higher with the PLMA. ${ }^{14}$ Both devices have a separate drainage tube that provides functional separation of the respiratory and gastrointestinal tracts when correctly positioned. The drainage tube was designed to reduce the risk of aspiration by avoiding gastric distension during positive pressure ventilation and by providing a conduit for regurgitated gastric contents. It also allows for insertion of an orogastric tube to aspirate stomach contents. Studies in anesthetized subjects and cadavers suggest that a properly positioned PLMA can reliably isolate the airway from fluids in the pharynx. ${ }^{15,16}$ There are also case reports that describe emission of intraoperative regurgitated fluid from the drainage tube with no airway soiling or aspiration, ${ }^{17,18}$ therefore supporting the usefulness of this tube in protecting against aspiration. However, it is crucial to position the 
device correctly so that the drainage tube is aligned with the esophagus. Aspiration of gastric contents was reported in a patient at low risk for aspiration when a PLMA was malpositioned with the drainage tube folded over during a laparoscopic cholecystectomy. ${ }^{19}$ In another report on non-obstetric patients, the authors described two cases of emission of regurgitated fluid from the drainage tube with no airway soiling and a third case in which aspiration occurred with the i-gel@, another supraglottic device with a drainage tube. ${ }^{20}$

The "gold standard" of general anesthesia for CD has been rapid sequence induction with tracheal intubation and cricoid pressure. However, the value of cricoid pressure as part of the rapid sequence induction technique has been challenged and debated. ${ }^{21}$ Does Yao et al.'s study support a change in practice regarding the choice of airway device in this patient population? The primary end point of the study was the incidence of aspiration. During general anesthesia for $\mathrm{CD}$, aspiration was reported to range from $1: 900^{22}$ to $1: 1547 .^{23}$ In an editorial, Pandit explained the Poisson distribution for quantifying the incidence of complications in an observational study including one group of patients and suggested that $>5,000$ patients are required to observe at least one case of aspiration with $>99 \%$ probability, assuming an incidence of aspiration with rapid sequence induction of no more than 1:1000. ${ }^{24}$ Yao's study is therefore underpowered for its primary end point. However, with the previous two series, ${ }^{5,6}$ we now have data on 4,643 low-risk women undergoing elective $\mathrm{CD}$ with an LMA with no documented cases of aspiration and one case of regurgitation. It is very important to point out though that the estimate of aspiration of 1:1000 likely includes patients at higher risk for aspiration, not the carefully selected lowrisk women included in the studies of LMA in CD in whom the incidence of aspiration is probably much lower. These factors would suggest that much more data are needed to ensure confidence in the safety of the LMA, even in Yao et al.'s select patient population which is not representative of obstetric patients receiving general anesthesia in our North American practice. The low overall incidence of aspiration also makes it unlikely that a randomized clinical trial will ever be conducted to address the issue of pulmonary aspiration with tracheal intubation $v s$ the LMA.

The use of the LMA as part of the failed intubation drill has saved lives. When correctly placed by an experienced user, the SLMA has advantages over the LMA Classic in terms of airway protection; therefore, it might be preferable in the obstetric population. We should continue to discourage repeated attempts at intubation and move earlier rather than later to a failed intubation drill. Insertion of the LMA at an early stage of the failed intubation drill may reduce the complications of repeated intubation attempts while providing adequate oxygenation and ventilation.
Practitioners might consider the use of the SLMA for slim fasted parturients with no gastroesophageal reflux who are scheduled for elective $\mathrm{CD}$ when there are particular risks associated with tracheal intubation. Otherwise, the standard for airway management in our obstetric patients should remain tracheal intubation. We should be careful about changing a technique that has been so successful in reducing airway-related maternal mortality.

\section{Est-il temps de reconsidérer l'intubation trachéale pour l'accouchement par césarienne?}

Des progrès significatifs ont été réalisés en anesthésie obstétricale. Le plus marquant a été une diminution impressionnante de la mortalité maternelle liée à l'anesthésie. Les Enquêtes confidentielles sur la mortalité maternelle ont débuté au Royaume-Uni en 1952 et dans chacun des dix premiers rapports triennaux, 30 à 50 décès étaient directement attribués à l'anesthésie. ${ }^{1}$ En revanche, les huit derniers rapports couvrant une période de 24 ans n'ont inclus que 41 décès directement liés à l'anesthésie, soit quasiment le nombre de décès qui survenaient auparavant au cours de chaque période de 3 ans. $^{2}$ Une évolution comparable a été observée en Amérique du Nord. Cette baisse de la mortalité liée à l'anesthésie est encore plus impressionnante si l'on considère le nombre croissant d'anesthésies administrées aux parturientes et l'augmentation continue du taux d'accouchement par césarienne au cours des 60 dernières années. Au Royaume-Uni, on a estimé que l'anesthésie pour césarienne était au moins 30 fois plus

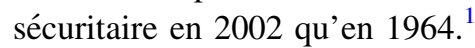

Il y a deux principales raisons à la survenue de ce remarquable succès. Tout d'abord, l'anesthésie générale pour césarienne est devenue beaucoup plus sûre et, ensuite, nous avons largement évité l'anesthésie générale en adoptant les techniques neuraxiales comme technique de référence pour la majorité des césariennes. La principale cause de décès dû à une anesthésie générale est liée à la prise en charge des voies aériennes. Au début, l'inhalation du contenu gastrique était le principal facteur responsable de mortalité maternelle. Un certain nombre de modifications ont été introduites au fil des ans pour résoudre ce problème, dont la technique d'induction à séquence rapide, qui inclut une pression cricoïdienne, une absence de ventilation au masque avant l'intubation trachéale et une utilisation de citrate de sodium et de bloqueurs $\mathrm{H}_{2}$. Cependant, la nécessité d'une intubation trachéale pour césarienne dans les années 1960 a introduit un autre problème: 
l'apparition d'échecs d'intubation trachéale et d'oxygénation inadaptée, ces facteurs ayant entraîné 34 décès maternels au Royaume-Uni au cours d'une période de neuf ans, de 1976 à $1984 .^{1}$ Ce type d'événements fatals est devenu rare grâce à l'amélioration de la formation, un meilleur monitorage avec l'utilisation de l'oxymétrie et de la capnographie, et une meilleure prise en charge des voies aériennes avec l'introduction d'exercices pour échec d'intubation ainsi que la plus grande disponibilité de dispositifs de secours des voies aériennes.

Le masque laryngé (Laryngeal Mask Airway ${ }^{\mathrm{TM}}$, LMA) est le principal dispositif de secours inclus dans les exercices pour échec d'intubation trachéale. Les comptes rendus et les séries de cas ont mis en évidence son utilisation bénéfique après l'échec d'une intubation trachéale dans les césariennes. ${ }^{3,4}$ En 2001, Han a décrit l'utilisation du LMA Classic ${ }^{\mathrm{TM}}$ chez 1067 parturientes subissant une césarienne programmée. ${ }^{5}$ Plus récemment, Halaseh et coll. ont décrit leur expérience du LMA ProSeal $^{\mathrm{TM}}$ (PLMA) chez 3000 femmes subissant la même intervention. ${ }^{6} \mathrm{Il} \mathrm{y}$ a eu des cas d'inhalation dans aucune de ces deux études, mais il y a eu un cas de régurgitation. ${ }^{6}$ Dans ce numéro du Journal, Yao et coll. apportent leur contribution à cette littérature par la description de l'utilisation du LMA Supreme ${ }^{\mathrm{TM}}$ (SLMA) chez 700 parturientes chinoises. ${ }^{7}$ Ils ont soigneusement sélectionné des patientes à faible risque avant de les inclure dans leur étude. Les femmes sont restées à jeun pendant au moins six heures en cas de chirurgie programmée et quatre heures dans les cas urgents; elles étaient exclues de l'étude en cas de difficultés d'intubation potentielles, d'indice de masse corporelle (IMC) $\geq 35 \mathrm{~kg} \cdot \mathrm{m}^{-2}$ ou de reflux gastro-œesophagien. Deux professionnels de la santé habitués à l'utilisation du SLMA ont inséré le dispositif chez toutes les patientes après induction de l'anesthésie et curarisation au rocuronium. Une prophylaxie antiacide était assurée par l'administration orale de citrate de sodium et de ranitidine. La pression sur le cricoïde était relâchée après confirmation d'une ventilation adaptée et le gonflement du ballonnet du SLMA. Un tube orogastrique prémonté était utilisé pour aspirer le contenu gastrique après l'insertion du SLMA et avant le réveil. Les auteurs ont réussi à insérer le SLMA dans tous les cas (98\% à la première tentative et $2 \%$ à la seconde tentative). Ils ont aussi réussi à mettre en place la sonde gastrique dans tous les cas et ont aspiré en moyenne $25 \mathrm{~mL}$ de sécrétions gastriques de $\mathrm{pH}$ moyen 2,2. Il n'y a pas eu de fuites décelables, d'épisodes de désaturation à $<92 \%$, et aucun signe de laryngospasme, de bronchospasme, de regurgitation ou d'inhalation. Moins de $4 \%$ des parturientes ont signalé un mal de gorge et il n'y a pas eu de différence en termes de résultats entre les césariennes programmées (576 cas) et les césariennes urgentes (124 cas).
Cette étude a été bien menée, mais il manque quelques détails utiles dans le compte rendu. Par exemple, les auteurs n'ont pas décrit les modifications hémodynamiques associées à l'insertion du SLMA, ce qui pourrait s'avérer un avantage par rapport à l'intubation trachéale. Quelques auteurs ont suggéré que la pression cricoïdienne pouvait empêcher l'insertion du LMA et la ventilation, mais ${ }^{8}$ Yao et coll. n'ont pas indiqué si le problème avait été rencontré ou si la pression sur le cricoïde avait dû être relâchée pour faciliter l'insertion et le bon positionnement du SLMA. Le $\mathrm{pH}$ du liquide gastrique recueilli était bas malgré la prophylaxie antiacide; cela pourrait être lié au moment sous-optimal de l'administration qui n'était pas précisé par les auteurs. La définition traditionnelle du risque d'inhalation (acide gastrique $>25 \mathrm{~mL}$, ou $0,4 \mathrm{~mL} \cdot \mathrm{kg}^{-1}$ et $\mathrm{pH}<2,5)$ pourrait suggérer que même certaines parturientes minces et à jeun courent un risque. Bien que certains auteurs aient été en faveur de l'utilisation de rocuronium à la place de la succinylcholine au cours des césariennes, ${ }^{9}$ la dose utilisée par Yao et coll. est inférieure à celle préconisée pour une induction à séquence rapide dans cette population de patientes, notamment là où le sugammadex est disponible, ${ }^{10}$ si bien que l'on pourrait s'attendre à un délai d'installation plus long qu'avec la succinylcholine. Ce choix pourrait avoir été influencé par la courte durée de l'intervention dans leur pratique. Cette plus faible dose pourrait être également appropriée pour prévenir la toux et les réflexes de lutte pendant l'insertion du SLMA avant de procurer les conditions adéquates d'intubation, mais Yao et coll. n'ont pas fourni d'information sur le délai écoulé entre l'induction et l'insertion du SLMA. On ignore également si les femmes ayant eu une césarienne étaient en travail ou avaient reçu des antalgiques morphiniques, qui, on le sait, ralentissent la vidange gastrique.

Comment doit-on interpréter les résultats de cette étude? Ces résultats s'appliquent-ils à notre pratique professionnelle? Nous devons effectivement souligner des différences significatives entre les césariennes réalisés dans cette étude et celles réalisés sous anesthésie générale en Amérique du Nord et dans de nombreuses régions d'Europe et d'Australie. Ainsi, par exemple, l'anesthésie générale est fréquemment employée dans l'hôpital de Yao, représentant $83 \%$ des césariennes effectuées au cours de l'étude d'une durée d'un an. Cela contraste vigoureusement avec nos habitudes actuelles où l'anesthésie générale est réservée à quelques situations d'urgence ou aux cas où le bloc a échoué, ou encore s'il existe une contre-indication à l'anesthésie régionale (une coagulopathie, par exemple). Certains centres ont signalé des taux d'anesthésie générale inférieurs à $1 \% .^{11}$ En conséquence, nos patientes pourraient ne pas être à jeun pendant quatre à six heures comme dans le cas de l'étude de Yao et coll. Il est 
également probable qu'elles souffrent et qu'elles auront reçu des antalgiques morphiniques, des médicaments connus pour augmenter le risque d'avoir un « estomac plein ». Les données démographiques de nos patientes sont également très différentes avec une prévalence croissante de l'obésité. Dans l'étude de Yao et coll., le poids moyen des patientes est de $65 \mathrm{~kg}$ avec un IMC de $26 \mathrm{~kg} \cdot \mathrm{m}^{-2}$, tandis que dans mon hôpital, les femmes ayant une césarienne ont un IMC de $33 \mathrm{~kg} \cdot \mathrm{m}^{-2} \cdot{ }^{12}$ Les auteurs font remarquer que les obstétriciens ont reçu pour consigne d'éviter toute pression excessive sur le fond utérin. Dans ma pratique, une pression excessive est fréquemment appliquée sur le fond utérin au cours de l'accouchement et je ne suis pas certain que nos obstétriciens puissent éviter, de façon prévisible, cette pratique. La pression sur le fond utérin peut entraîner une régurgitation, ce qui pourrait constituter un risque d'inhalation. En fait, le cas de régurgitation dans la série de cas précédente impliquant l'utilisation de PLMA est survenue à la suite d'une pression sur le fond utérin au cours de l'accouchement. ${ }^{6}$ Dans l'étude publiée dans ce numéro du Journal, le SLMA a été inséré par deux professionnels expérimentés dans les conditions optimales de relâchement musculaire et son bon positionnement avait été confirmé. En conséquence, ces constatations pourraient ne pas s'appliquer aux cas pour lesquels le SLMA est inséré par des professionnels moins expérimentés, une situation fréquente dans nos établissements d'enseignement. L'audit publié récemment au Royaume-Uni par le Royal College of Anaesthetists et la Difficult Airway Society a cité l'obésité et le recours aux professionnels novices comme facteurs intervenant dans les complications majeures des voies aériennes avec des dispositifs supraglottiques. ${ }^{13}$ Dans l'étude de Yao et coll., la durée de l'intervention chirurgicale a également été plus courte que celle que l'on connaît dans beaucoup d'hôpitaux universitaires. En conséquence, la population de patientes et les pratiques de cet hôpital chinois sont très différentes de celles rencontrées en Amérique du Nord où les femmes recevant une anesthésie générale pour césarienne présentent un risque beaucoup plus élevé de régurgitation et d'inhalation.

Le SLMA a été introduit en 2007 comme une version modifiée à usage unique du PLMA. Les études ont indiqué que le taux de succès, le délai d'insertion et les complications sont comparables entre les deux dispositifs, mais que la pression de fuite buccopharyngée est plus élevée avec le PLMA. ${ }^{14}$ Les deux dispositifs comportent un tube de drainage distinct qui procure une séparation fonctionnelle des voies respiratoires et du tube digestif quand il est correctement positionné. Le tube de drainage a été conçu pour réduire le risque d'inhalation en évitant une distension gastrique au cours de la ventilation en pression positive et en fournissant une évacuation au contenu gastrique régurgité. Il permet également l'insertion d'un tube orogastrique pour l'aspiration du contenu gastrique. Les études menées ches des sujets anesthésiés et sur des cadavres suggèrent qu'un PLMA correctement placé peut isoler de façon fiable les voies aériennes des liquides présents dans le pharynx. ${ }^{15,16}$ Certains cas ont également été décrits avec émission de liquide régurgité en peropératoire par le tube de drainage, sans souillure ni inhalation dans les voies aériennes, confortant ainsi l'intérêt de ce tube pour protéger contre l'inhalation. ${ }^{17,18} \mathrm{Il}$ est cependant essentiel de positionner correctement le dispositif de façon à ce que le tube de drainage soit dans l'alignement de l'œsophage. Une inhalation du contenu gastrique a été signalée chez un patient à faible risque d'inhalation au cours d'une cholécystectomie par laparoscopie quand un PLMA a été mal positionné, le tube de drainage étant plié. ${ }^{19}$ Dans d'autres comptes rendus chez des patients non obstétricaux, des auteurs ont décrit deux cas d'émission de liquide régurgité par le tube de drainage, sans souillure des voies aériennes, et un troisième cas chez lequel une inhalation est survenue avec l'i-gel $\odot$, un autre dispositif supraglottique avec tube de drainage. ${ }^{20}$

$\mathrm{La}$ « référence » pour l'anesthésie générale en cas de césarienne a été l'induction à séquence rapide avec intubation trachéale et pression cricoïdienne. Cependant, la contribution de la pression cricoïdienne dans le cadre de la technique d'induction à séquence rapide a été remise en cause et discutée. ${ }^{21}$ L'étude de Yao et coll. est-elle en faveur d'une modification de la pratique concernant le choix d'un dispositif pour voies aériennes dans cette population de patientes? Le critère d'évaluation principal de l'étude était l'incidence d'inhalation. Au cours de l'anesthésie générale pour césarienne, le taux d'inhalation est compris entre $1: 900^{22}$ et $1: 1547$, selon les études. ${ }^{23}$ Dans une étude observationnelle incluant un groupe de patients, Pandit a expliqué la distribution de Poisson pour la quantification de l'incidence des complications et a suggéré qu'il était nécessaire d'avoir plus de 5000 patients pour observer au moins un cas d'inhalation avec une probabilité $>99 \%$, en supposant que l'incidence d'inhalation avec une induction à séquence rapide n'est pas supérieure à 1:1000. ${ }^{24}$ L'étude de Yao n'a donc pas la puissance suffisante pour ce critère d'évaluation principal. Cependant, avec les deux séries précédentes, nous disposons maintenant de données sur 4643 femmes à faible risque subissant une césarienne programmée avec un LMA sans cas documenté d'inhalation et un cas de régurgitation. ${ }^{5,6} \mathrm{Il}$ est néanmoins très important de souligner que l'estimation d'un cas d'inhalation sur 1000 inclut probablement des patientes à risque plus élevé et non les femmes à faible risque d'inhalation soigneusement sélectionnées qui sont incluses dans les études du LMA dans la césarienne chez lesquelles l'incidence d'inhalation est probablement beaucoup plus faible. Ces facteurs 
suggéreraient qu'il faut beaucoup plus de données pour garantir la confiance dans l'innocuité du LMA, même dans la population de patientes sélectionnées de Yao et coll., qui n'est pas représentative des patientes enceintes recevant une anesthésie générale en Amérique du Nord. L'incidence globalement faible d'inhalation rend également improbable la réalisation d'un essai clinique randomisé qui aborderait le problème de l'inhalation pulmonaire en comparant l'intubation trachéale et le LMA.

L'utilisation du LMA dans le cadre d'exercices d'échec d'intubation a sauvé des vies. Quand il est correctement positionné par un utilisateur expérimenté, le SLMA présente des avantages par rapport au LMA Classic en termes de protection des voies aériennes; il pourrait donc être préférable dans la population obstétricale. Nous devons continuer à décourager des tentatives répétées d'intubation et passer plus rapidement que tardivement à un exercice d'échec d'intubation. L'insertion du LMA au stade précoce de l'exercice d'échec d'intubation peut réduire les complications liées aux tentatives répétées d'intubation, tout en assurant une oxygénation et une ventilation appropriées. Les praticiens pourraient envisager l'utilisation du SLMA chez des parturientes minces et à jeun, sans reflux gastro-œsophagien, qui ont une césarienne programmée, en présence de risques particuliers associés à une intubation trachéale. Dans les autres cas, la norme de prise en charge des voies aériennes en obstétrique doit rester l'intubation trachéale. Nous devons faire preuve de prudence avant de changer une technique qui a tellement réussi à réduire la mortalité maternelle liée aux voies aériennes.

Conflicts of interest None declared.

Funding This article was supported solely by departmental funds.

\section{References}

1. Cooper GM, McClure JH. Maternal deaths from anaesthesia. An extract from Why Mothers Die 2000-2002, the Confidential Enquiries into Maternal Deaths in the United Kingdom: Chapter 9: Anaesthesia. Br J Anaesth 2005; 94: 417-23.

2. Cantwell R, Clutton-Brock T, Cooper G, et al. Saving Mothers' Lives: Reviewing maternal deaths to make motherhood safer: 2006-2008. The Eighth Report of the Confidential Enquiries into Maternal Deaths in the United Kingdom. BJOG 2011; 118(Suppl 1): $1-203$

3. McDonnell NJ, Paech MJ, Clavisi OM. ANZCA Trials Group. Difficult and failed intubation in obstetric anaesthesia: an observational study of airway management and complications associated with general anaesthesia for caesarean section. Int J Obstet Anesth 2008; 17: 292-7.

4. Awan R, Nolan JP, Cook TM. Use of a ProSeal laryngeal mask airway for airway maintenance during emergency caesarean section after failed tracheal intubation. Br J Anaesth 2004; 92 : 144-6.
5. Han TH, Brimacombe J, Lee EJ, Yang HS. The laryngeal mask airway is effective (and probably safe) in selected healthy parturients for elective cesarean section: a prospective study of 1067 cases. Can J Anesth 2001; 48: 1117-21.

6. Halaseh BK, Sukkar ZF, Hassan LH, Sia AT, Busnag WA, Adarbeh $H$. The use of ProSeal laryngeal mask airway in caesarean section-experience in 3000 cases. Anaesth Intensive Care 2010; 38: 1023-8.

7. Yao WY, Li SY, Sng BL, Lim Y, Sia AT. The LMA Supreme ${ }^{\mathrm{TM}}$ in 700 parturients undergoing cesarean delivery: an observational study. Can J Anesth 2012; 59: this issue. DOI:10.1007/s12630-0129718-4

8. Aoyama K, Takenaka I, Sata T, Shigematsu A. Cricoid pressure impedes positioning and ventilation through the laryngeal mask airway. Can J Anaesth 1996; 43: 1035-40.

9. Sharp LM, Levy DM. Rapid sequence induction in obstetrics revisited. Curr Opin Anaesthesiol 2009; 22: 357-61.

10. Williamson RM, Mallaiah S, Barclay P. Rocuronium and sugammadex for rapid sequence induction of obstetric general anaesthesia. Acta Anaesthesiol Scand 2011; 55: 694-9.

11. Palanisamy A, Mitani AA, Tsen LC. General anesthesia for cesarean delivery at a tertiary care hospital from 2000 to 2005: a retrospective analysis and 10-year update. Int J Obstet Anesth 2011; 20: 10-6.

12. Muir HA, Peterson-Layne C, Allen TK, Habib AS. Influence of Obesity on Need for Conversion of Neuraxail to General Anesthesia for Cesarean Section. American Society of Anesthesiologists Meeting 2009; A 214.

13. Cook TM, Woodal N, Freck C. 4th National Audit Project of The Royal College of Anaesthetists and The Difficult Airway Society. Major Complications of Airway Management in the UK. Report and Findings, March 2011. Available from URL: http://rcoa.ac. uk/nap4 (accessed April 2012).

14. Wong DT, Yang JJ, Jagannathan N. Brief review: The LMA Supreme $^{\mathrm{TM}}$ supraglottic airway. Can J Anesth 2012; DOI: 10.1007/ s12630-012-9673-0.

15. Keller C, Brimacombe J, Kleinsasser A, Loeckinger A. Does the ProSeal laryngeal mask airway prevent aspiration of regurgitated fluid? Anesth Analg 2000; 91: 1017-20.

16. Evans NR, Gardner SV, James MF. ProSeal laryngeal mask protects against aspiration of fluid in the pharynx. $\mathrm{Br} \mathrm{J}$ Anaesth 2002; 88: 584-7.

17. Evans NR, Llewellyn RL, Gardner SV, James MF. Aspiration prevented by the ProSeal laryngeal mask airway: a case report. Can J Anesth 2002; 49: 413-6.

18. Su BC, Yang MW, Lee HC, Chang CH, Lin CC. Protection against large-volume regurgitated fluid aspiration by the ProSeal laryngeal mask airway. Acta Anaesthesiol Taiwan 2008; 46: 34-8.

19. Brimacombe J, Keller C. Aspiration of gastric contents during use of a ProSeal laryngeal mask airway secondary to unidentified foldover malposition. Anesth Analg 2003; 97: 1192-4.

20. Gibbison B, Cook TM, Seller C. Case series: Protection from aspiration and failure of protection from aspiration with the i-gel airway. Br J Anaesth 2008; 100: 415-7.

21. Neilipovitz DT, Crosby ET. No evidence for decreased incidence of aspiration after rapid sequence induction. Can J Anesth 2007; 54: 748-64.

22. Soreide E, Bjornestad E, Steen PA. An audit of perioperative aspiration pneumonitis in gynaecological and obstetric patients. Acta Anaesthesiol Scand 1996; 40: 14-9.

23. Dindelli $M$, La Rosa $M$, Rossi $R$, et al. Incidence and complications of the aspiration of gastric contents syndrome during cesarean section in general anesthesia (Italian). Ann Ostet Ginecol Med Perinat 1991; 112: 376-84.

24. Pandit JJ. Gambling with ethics? A statistical note on the Poisson (binomial) distribution. Anaesthesia 2008; 63: 1163-6. 